\title{
Der Palmengarten im Jahr 2016
}

\section{Matthias Jenny \& Manfred Wessel}

Auch wenn im Winter die Natur im Freien ruht, verwandelt sich der Palmengarten in der dunklen Jahreszeit in einen ganz besonderen, magischen Ort, an dem man außer in den Gewächshäusern auch draußen Spannendes erleben kann. Der Winter 2015/2016 ist wieder viel zu warm und es gibt auch im Januar keinen Schnee. Dafür glitzern an allen Ecken seit Dezember die Winterlichter. Ein neuer Rundgang bezieht diesmal den Bereich des Sommer-Sukkulentengartens, die zum Winterschutz verpackten Palmen, das Haus Leonhardsbrunn sowie die östliche Fassade des Gesellschaftshauses mit ein. Auch dieses Mal ist ein neues leuchtendes Schneeglöckchen dazugekommen. Erneut wurde ein Rekord der Winterlichter-Besucher aufgestellt. Der Palmengarten hofft, die Besucherzahlen auch in Zukunft steigern zu können, vor allem im Winter. Dazu soll auch der Bau des Blüten- und Schmetterlings-

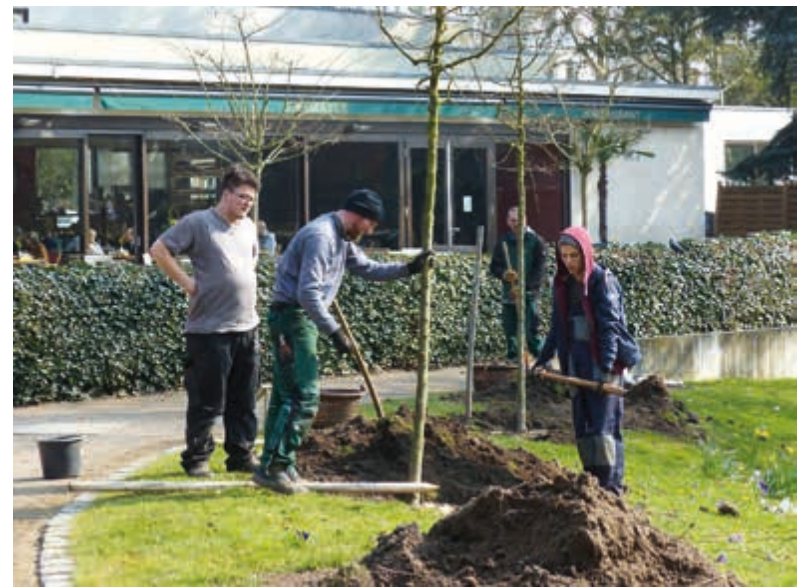

Abb. 1: Die Linden in der Nähe des kleinen Weihers werden durch Zier-Apfelbäume ersetzt.

hauses beitragen, in dem vor allem im Winter frei fliegende tropische Schmetterlinge gezeigt werden. Als Vorbereitung dazu werden das alte Blütenhaus und die angrenzenden maroden Gewächshäuser

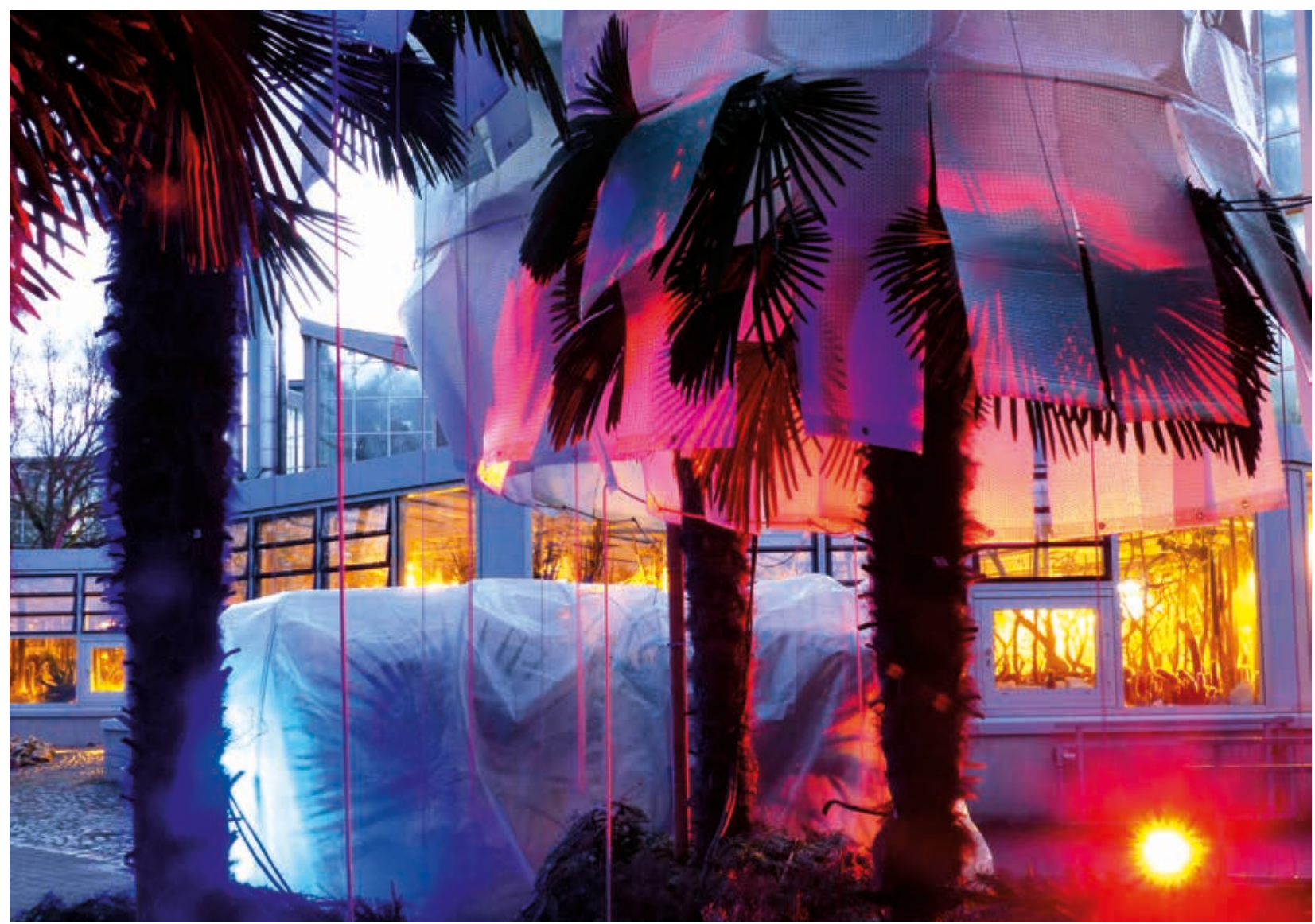

Abb. 2: Die mit Winterschutz versehenen Palmen leuchten während der Winterlichter. 

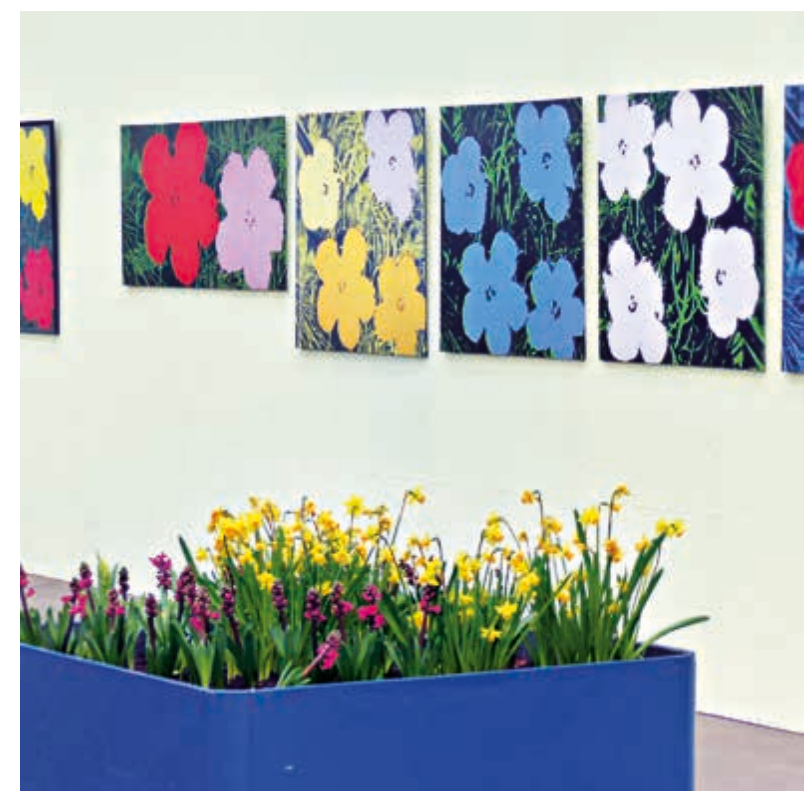

Abb. 3: Frühlingsblumenausstellung mit Blumen von WARHOL.

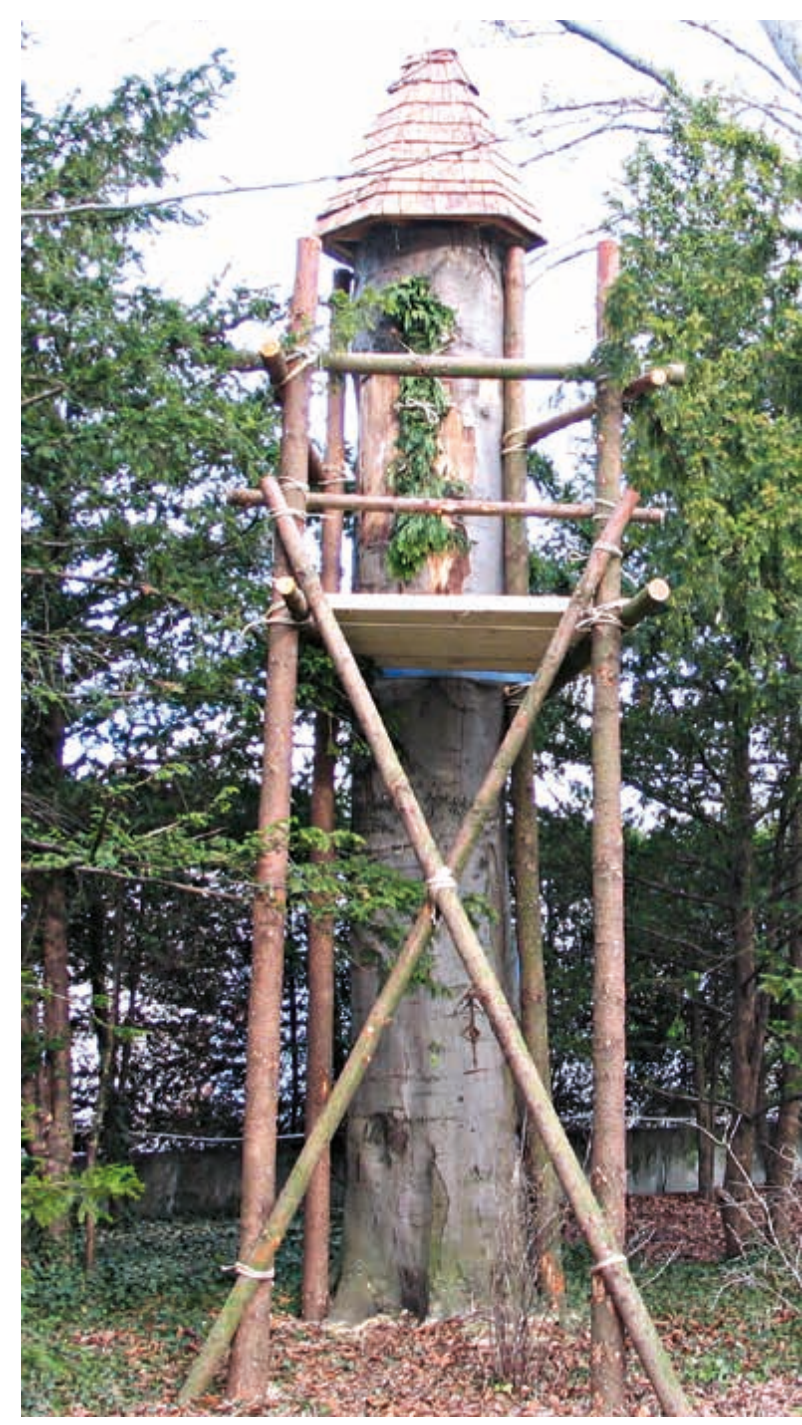

Abb. 4: Zeidlerbaum-Aktion im Botanischen Garten. der Gärtnerei abgerissen. Die Vorbereitungen für den Frühlingsball laufen auf Hochtouren, Stargast ist dieses Jahr Wencke Myнre. Jedes Jahr schon lange erwartet wird die Kamelien-Ausstellung in der Galerie West, die erste Blumenschau der Saison. Aufgrund der relativ hohen Temperaturen der vorangegangenen Wochen sind bereits zur Eröffnung besonders viele Blüten geöffnet.

Bereits am 8. Januar treffen sich 40 unentwegte Besucherinnen und Besucher des Botanischen Gartens wie gewohnt zum sonntäglichen Winterspaziergang. Der Jahreszeit angemessene Temperaturen beschränkt die Zahl der blühenden Pflanzen, aber schön ist es trotzdem, den Botanischen Garten zu erleben.

Seit Anfang des Jahres nimmt die Bus-Linie 36 eine andere Route durch das Westend und spart die Haltestelle am Palmengarten aus. Das führt ab Februar zu Irritationen und Unmut. Ein Garten und sein Pflanzenbestand sind stets im Wandel begriffen. Im Palmenhaus ist eine stattliche Washingtonia-Palme mittlerweile so hoch gewachsen, dass sie bis an das Glasdach reicht und dieses auf Dauer zerstören würde. Sie muss deshalb leider gefällt werden. Ein Stück des Stammes wird aber als Exponat für die Palmen-Ausstellung 2018 aufgehoben. In der Galerie Ost wird mit der Frühlingsblumen-Ausstellung die Blütezeit klassischer Frühjahrsgeophyten wie Narzissen, Tulpen und Traubenhyazinthen im Vergleich zum Freiland vorverlegt. Mit Warhols Bildern (Artist's Proof) wird das Thema der natürlichen und industriellen Reproduktion gegenübergestellt.

Am letzten Sonntag im Februar öffnet der Botanische Garten nach der Winterpause seine Pforten und startet in die neue Saison. Eine Führung mit über 100 Menschen verdeutlicht die schon weit fortgeschrittene Vegetationsentwicklung und bringt den Technischen Leiter gehörig ins Schwitzen.

Die Frühlingsblumen-Ausstellung endet am ersten März-Wochenende, an dem auch der Frühlingsball stattfindet. Die Erlöse kommen dem Blüten- und Schmetterlingshaus zugute. 


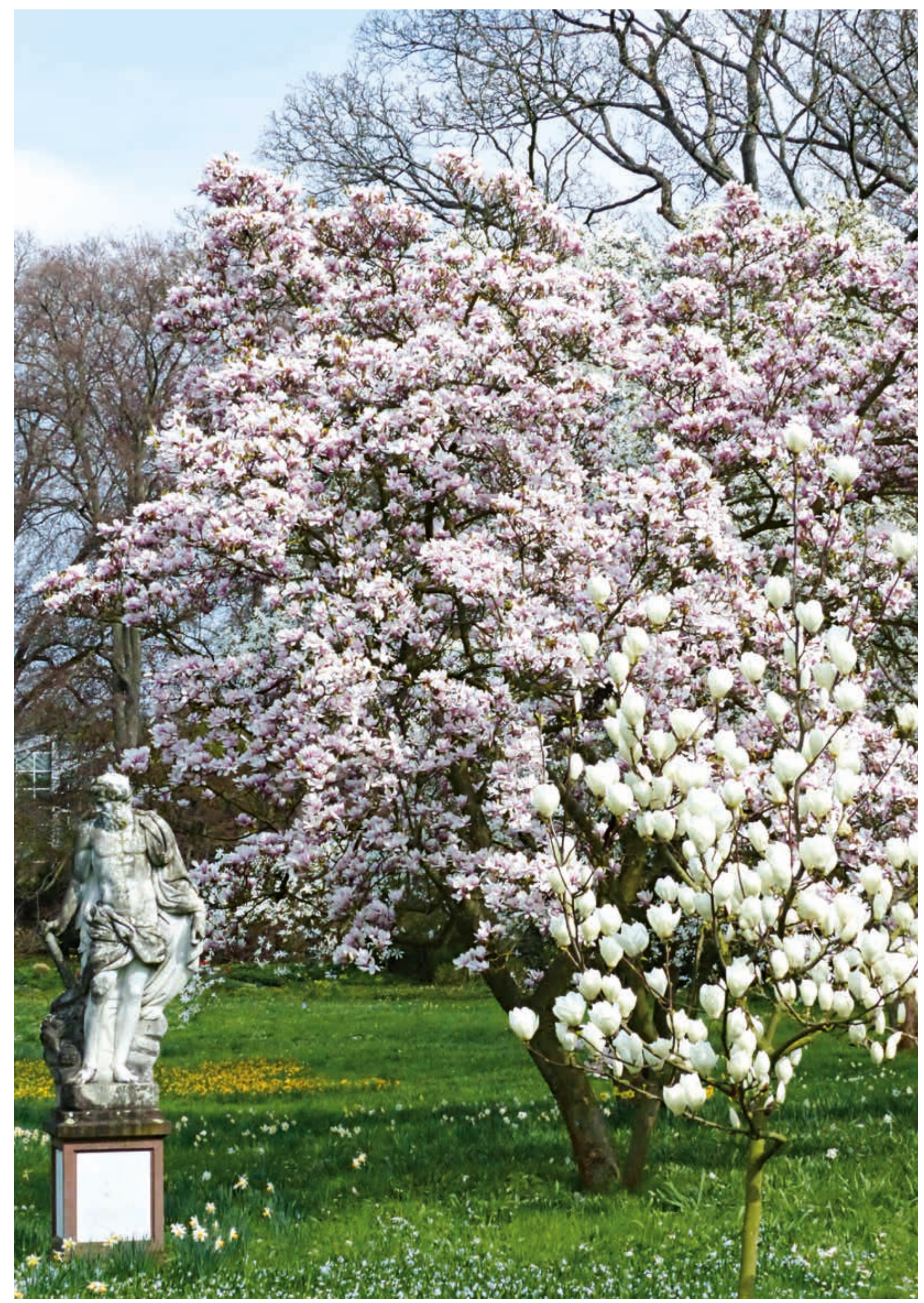

Abb. 5: Aufgrund des vergangenen sehr milden Winters blühen die Magnolien besonders üppig. 


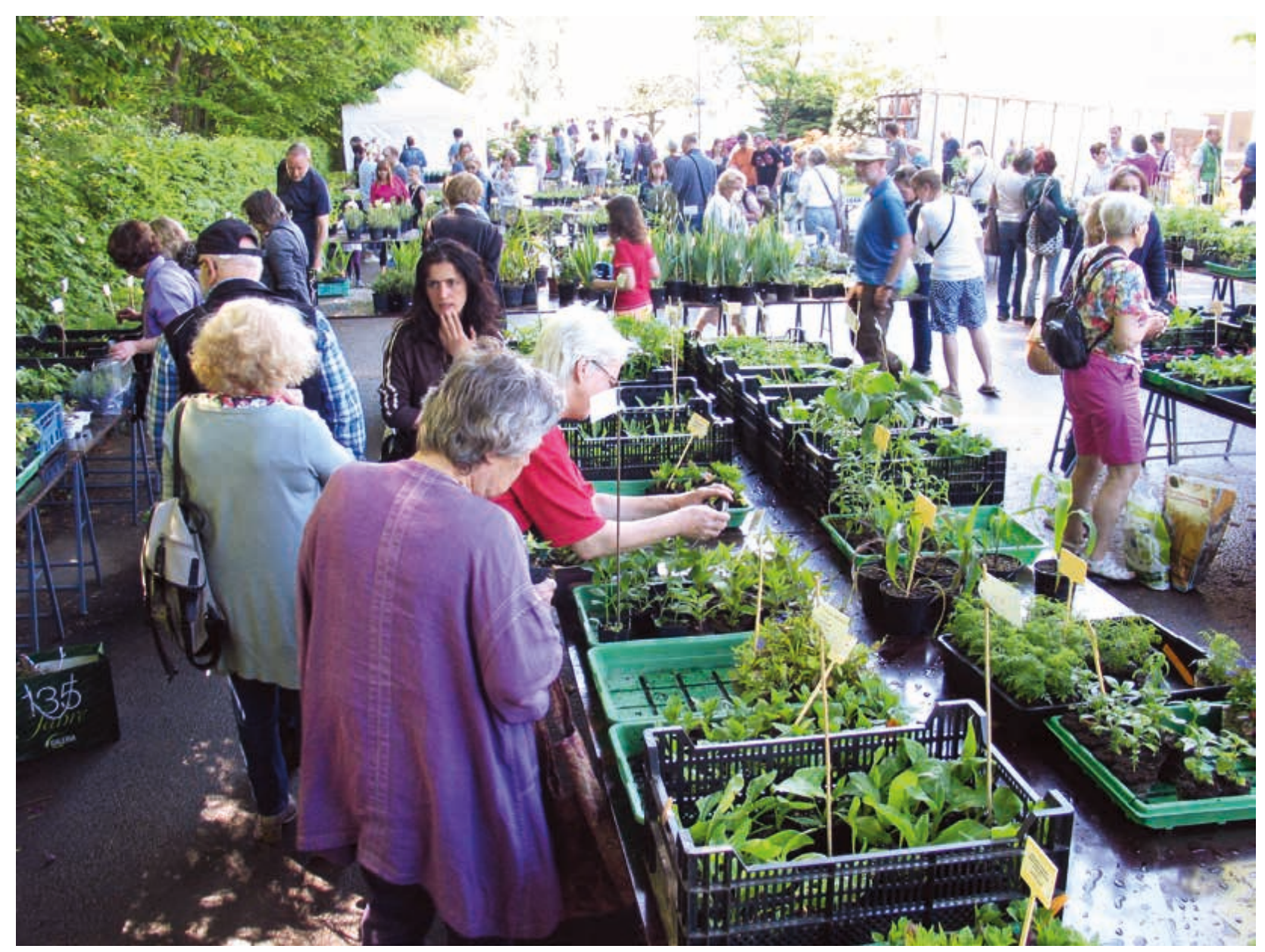

Abb. 6: Pflanzenbörse im Mai im Botanischen Garten.

Der Blumenschmuck ist diesmal in Gelb gehalten. Der März ist für viele der Start in eine neue Gartensaison. Dementsprechend ist der Zulauf bei der Grünen Börse groß. Garten- und Pflanzenfreunde decken sich dabei auf der Raritätenbörse mit grünen Schätzen ein. In der Galerie Ost gibt es jede Menge Informationen aus dem grünen Bereich. BioFrankfurt und Partner präsentieren sich unter dem Motto „Artenvielfalt bewegt“. Gleichzeitig findet die Orchideen-Verkaufsausstellung statt, die ebenfalls jedes Jahr viele Pflanzenbegeisterte anzieht. Die Villa Leonhardi erhält ein neues Nutzungskonzept mit Ausstellungen und Veranstaltungen und ein neuer Betreiber namens Goose bietet den Gartenbesuchern verschiedene Waffelkreationen an.

Im März wird im Botanischen Garten der sogenannte Zeidler-Baum seinem Zwecke zugeführt. Eine abgestorbene, auf $5 \mathrm{~m}$ gestutzte Rot-Buche wird nach Zeidler-Art für eine traditionelle und ursprüngliche Form der Imkerei hergerichtet. Die- se Aktion zusammen mit der Gruppe der „Bienenbotschaft" erstreckt sich über drei Tage und wird vom Gartenpublikum und der Presse mit viel Interesse verfolgt.

Im April blüht es im Palmengarten im Freien sowie unter Glas sehr üppig. Besonders spannend ist die im Tropicarium blühende Riesen-Bromelie Alcantarea imperialis. Viele Besucher fragen nach ihr. Bunt ist es auch rund um das Osterhasengärtchen, in dem traditionell Kinder Ostereier sammeln dürfen, und immer wieder aufs Neue begeistern die leuchtend pink-violetten Azaleen in der Galerie West.

Im Mai blüht schließlich auch der Rhododendron-Garten am Großen Weiher. Die Energie für den Palmengarten und dementsprechend auch für das Klima in den Gewächshäusern, das für ein gutes Gedeihen der Pflanzen nötig ist, kommt zukünftig als Fernwärme in den Garten. Das gasbetriebene Blockheizkraftwerk des Palmengartens wird 


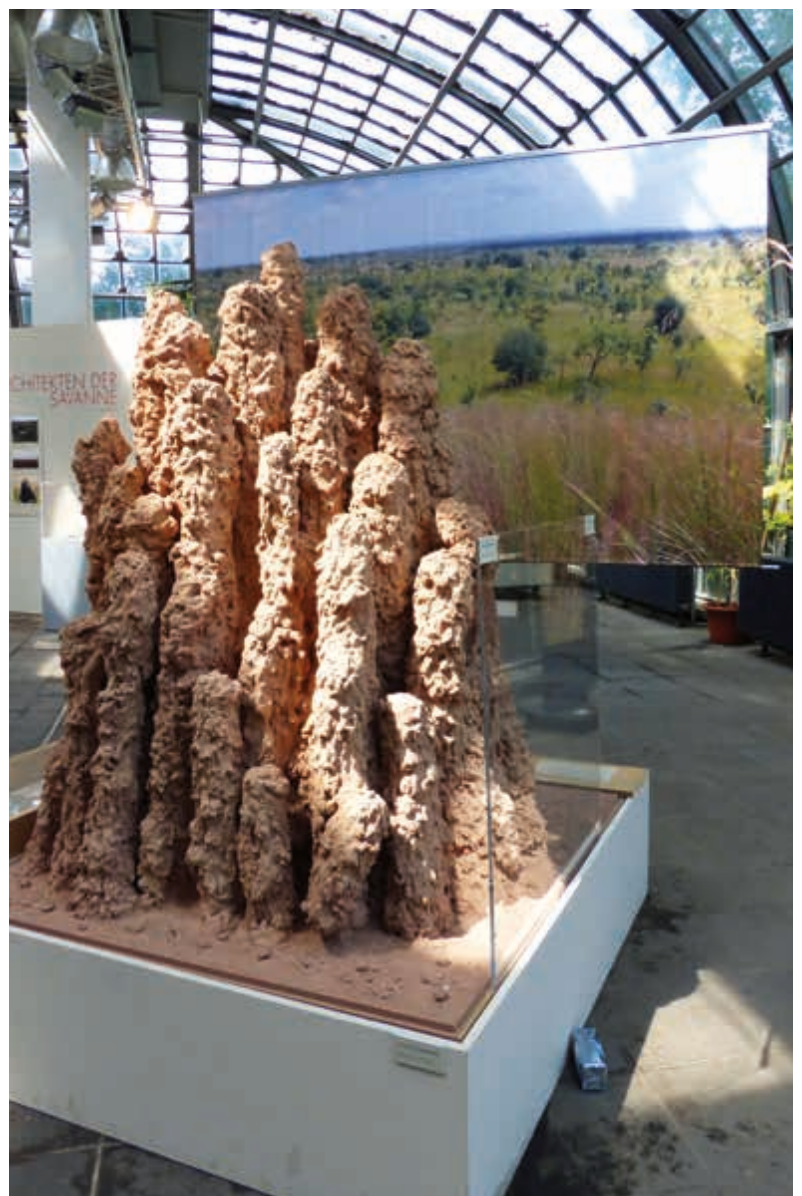

Abb. 7: Termitenhügel in der Savannenausstellung.

zugunsten eines Fernwärmeanschlusses aufgegeben. Die Wärme liefern nun das Fernwärmenetz der Mainova sowie das Müllheizkraftwerk in der Nordweststadt. Ab Mai dreht sich in diesem Sommer in der großen Informationsausstellung „Lebensraum Savanne" alles um afrikanische Savannen. Die Vielfalt der Pflanzen und Tiere, ihre Wechselbeziehungen, die Savanne als Wiege der Menschheit und die Nutzung der Savannenpflanzen durch den Menschen werden thematisiert. Besonders beeindruckende Exponate sind ein vom Senckenberg-Museum naturgetreu nachgebauter Termitenhügel sowie ein originales transportables „Leichtbauhaus“ der nomadischen Fulbe-Ethnie. Ausstellung und Rahmenprogramm finden guten Zuspruch.

Anfang Mai findet im Botanischen Garten die stark geschätzte Pflanzenbörse statt. Aus alter Verbundenheit beteiligt sich auch der Wissenschaftsgarten der Universität mit Pflanzen sowie Mitarbeiterinnen und Mitarbeitern an dieser wie immer sehr gut besuchten Veranstaltung. Die vie-

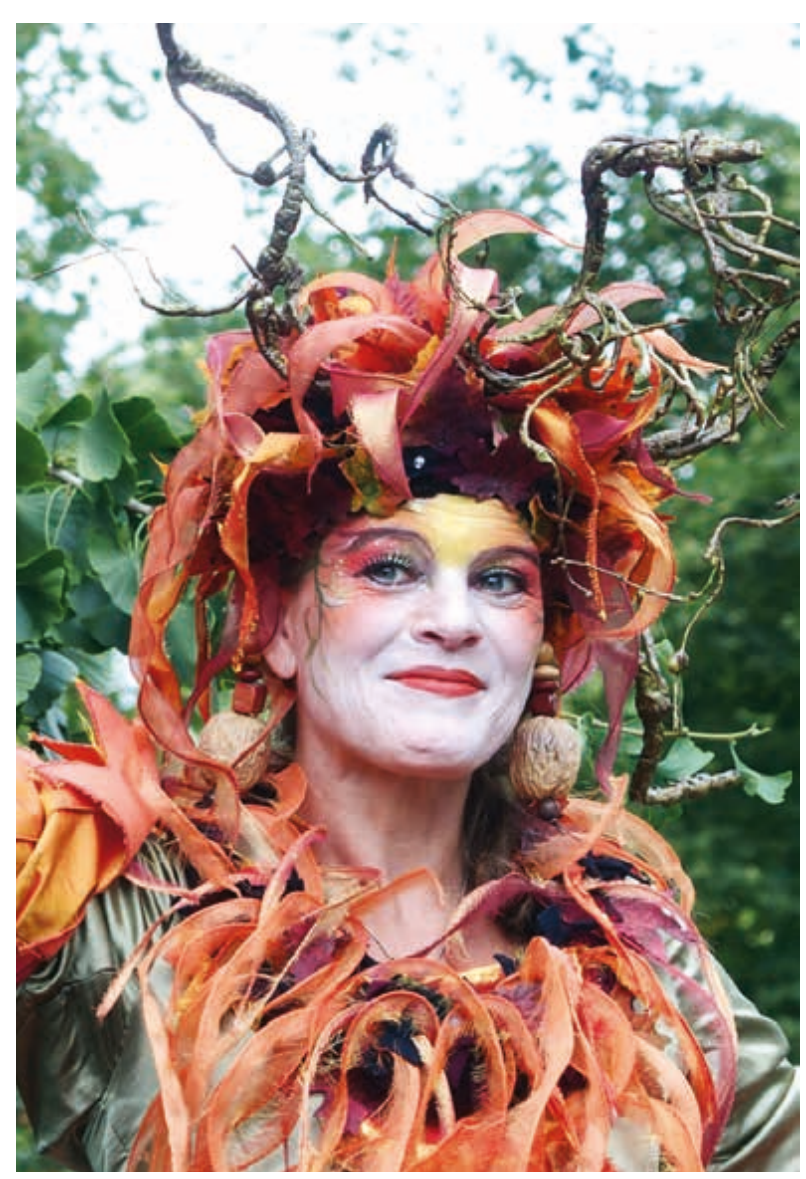

Abb. 8: Wilder Sonntag mit Geschichten, Wissenswertem, Gedichten und Musik zum Thema Bäume.

len Gäste an diesem Tag zeigen ihre Verbundenheit mit dem Botanischen Garten, sodass auch die beschäftigten Mitarbeiterinnen und Mitarbeiter und die Helferschar des Freundeskreises viel Freude an diesem Tag haben. Ende Mai werden zusammen mit einem Urenkel des Naturforschers, Arztes und Japanreisenden Philipp Franz von Siebold die "Siebold-Beete“ eröffnet und zugleich ein Siebold-Lehrpfad durch die Ostasien-Abteilung präsentiert.

Der Juni gibt sich ziemlich kalt und verregnet. Zum Rosen- und Lichterfest ist das Wetter aber passabel, so dass das größte Fest des Palmengartens wieder ein voller Erfolg wird. Am Samstag-Abend herrscht Picknickstimmung und werden auf den Wiesen leuchtende Muster aus unzähligen Teelichtern gelegt. Den Abschluss des Abends bildet ein 15-minütiges Feuerwerk, das am großen Weiher abgebrannt wird. Die Rosenausstellung steht ganz im Zeichen des 400. Todestages von William Shakespeare. Die vom Verband Botanischer 


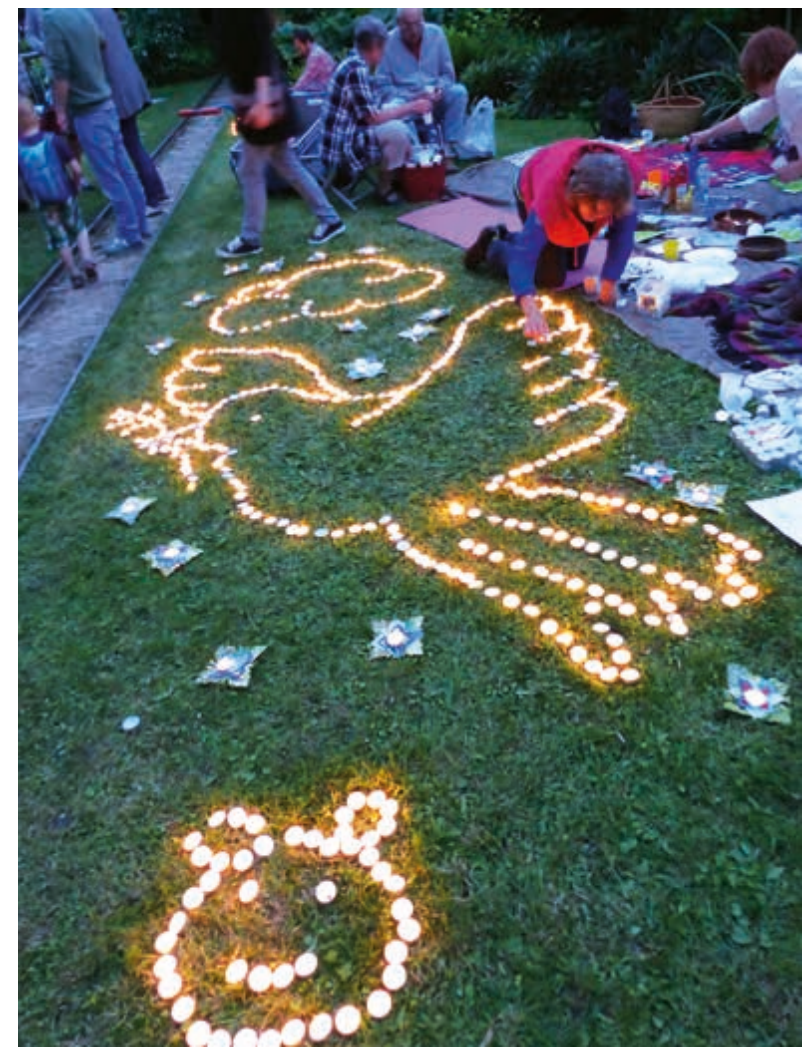

Abb. 9: Teelichter-Bild während des Rosen- und Lichterfestes, dem größten Fest im Palmengarten.

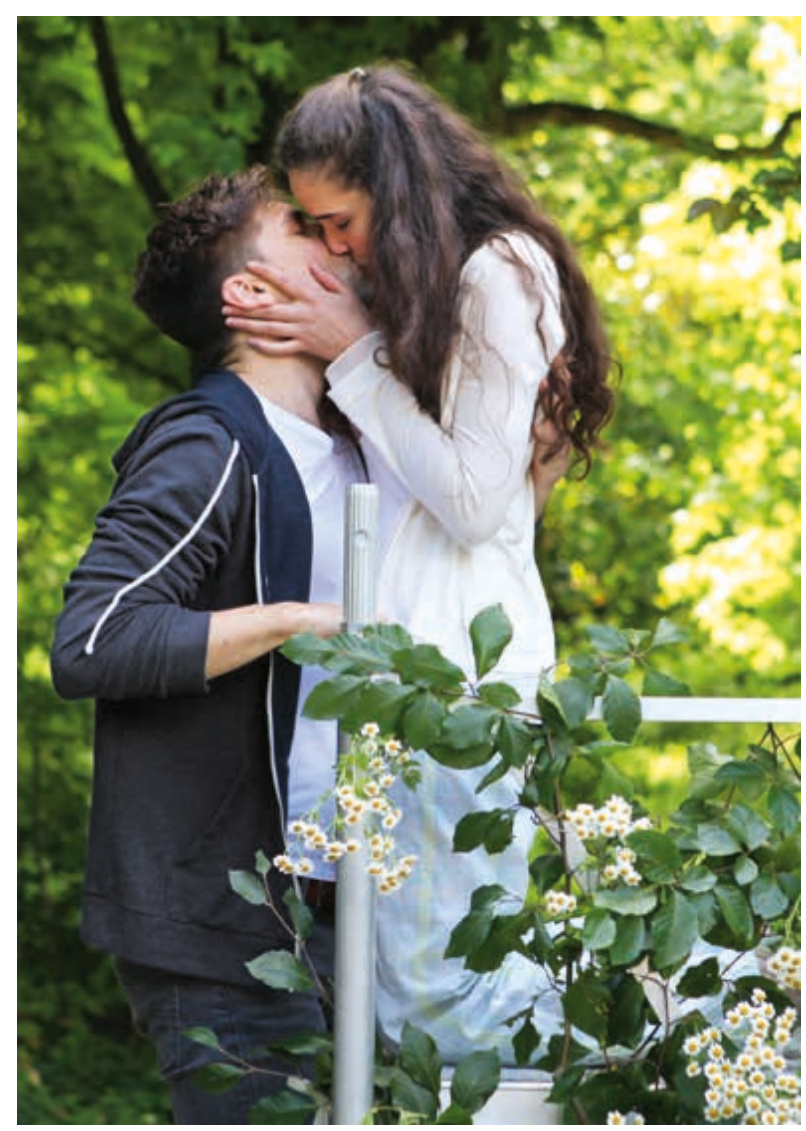

Abb. 10: Der Kuss von Romeo und Julia während der Shakespeare-Aufführung im Botanischen Garten.
Gärten organisierte „Woche der Botanischen Gärten" mitsamt Ausstellung widmet sich aus Anlass des 400. Todestages des großen Barden der Aufgabe, sein Werk und seine Beziehungen zur Pflanzenwelt darzustellen. Der Botanische Garten zeigt die von Stefan Schneckenburger, ehemaliger Kustos im Palmengarten, erstellte Ausstellung, die im gesamten Garten verteilt auf bestimmte Pflanzen verweist und die sein Leben und Schaffen ausführlich erläutert. Der Höhepunkt ist aber sicherlich das Angebot einer Theateraufführung. „A LoFT Frankfurt" präsentiert in vier öffentlichen englischsprachigen Auftritten "Shakespeare in the Garden presents Hortus Inclusus“. An insgesamt acht verschiedenen Standorten im Garten werden Szenen aus acht Stücken Shakespeares dargeboten. Annähernd 400 Personen folgen den Schauspielern durch den Garten und sind vom Gebotenen und von der besonderen Atmosphäre der Botanischen Garten-Bühne hellauf begeistert. Der Botanische Garten wird durch dieses Angebot von vielen neuen Gästen entdeckt.

Immer wieder diskutiert wird die Zukunft des Areals zwischen Palmengarten, Botanischem Garten und Grüneburgpark sowie die spätere Nutzung der leerstehenden Institutsgebäude. Noch ist nichts konkret, eine Meldung geht von einer Wohnbebauung sowie der Errichtung einer Grundschule aus. Der Jugendverkehrsgarten soll umziehen. Der Sommer im Palmengarten bietet eine Fülle von Veranstaltungen für jeden Geschmack wie die Musikreihen, das Kinder-Ferienprogamm der Grünen Schule, das GWH-Familienfest sowie das BILD-Sommerfest.

Im Juli genießen viele Besucherinnen und Besucher das sommerliche Ambiente des Palmengartens. Die Räume der Villa Leonhardi sind in verschiedenen Farben kunterbunt gestrichen. Außer mit dem kulinarischen Angebot soll die Villa auch durch etwas unkonventionelle Ausstellungen wie z. B. „Blümchensex“ Besucher anlocken. An einem Konzept für die Villa wird weiter gearbeitet. So werden im Sommer z. B. auch Yoga-Kurse angeboten. Bis in den August hinein zeigen die Arbeiten der Kölner Malerin Bettina Mauel in der Galerie Ost Licht und Farbe im Garten. 


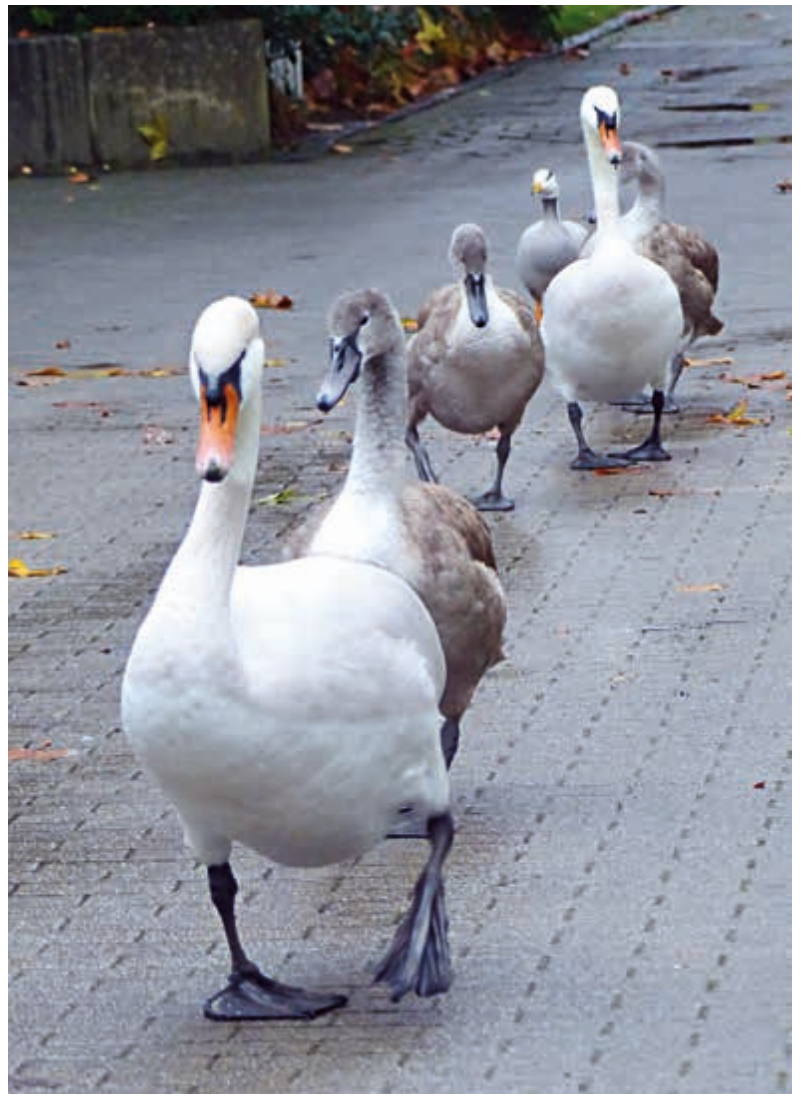

Abb. 11: „Schwanenmarsch“.
Der August ist nach einem eher verregneten Sommer noch einmal schön warm, so dass die Freunde unserer Freiluftveranstaltungen auf ihre Kosten kommen, ob es nun die Konzerte sind oder die Dezernatsveranstaltung „Wilder Sonntag", bei dem diesmal heimische und exotische Bäume literarisch vorgestellt werden. Gut angenommen wird auch die abendliche Führungsreihe „Pflanzen mit Migrationshintergrund“. Unter anderem inspiriert uns dies, in Kooperation mit BioFrankfurt für 2017 eine Informationsausstellung über Neophyten, also Pflanzen mit Migrationshintergrund, auszuarbeiten. Ganz allgemein sind neue Ball-Konzepte gefragt, und auch der Frühlingsball im Palmengarten soll ein neues Gesicht bekommen.

Der September zeigt sich meist in leuchtenden Gelb-Rottönen: Die Dahlien vor dem Haus Leonhardsbrunn kündigen den Herbst an, auf dem Herbstfest und der Erntedankschau sind diverse Früchte der Saison und Herbstblumen zu sehen. Für die Kleinen ist es jedes Jahr ein Highlight,

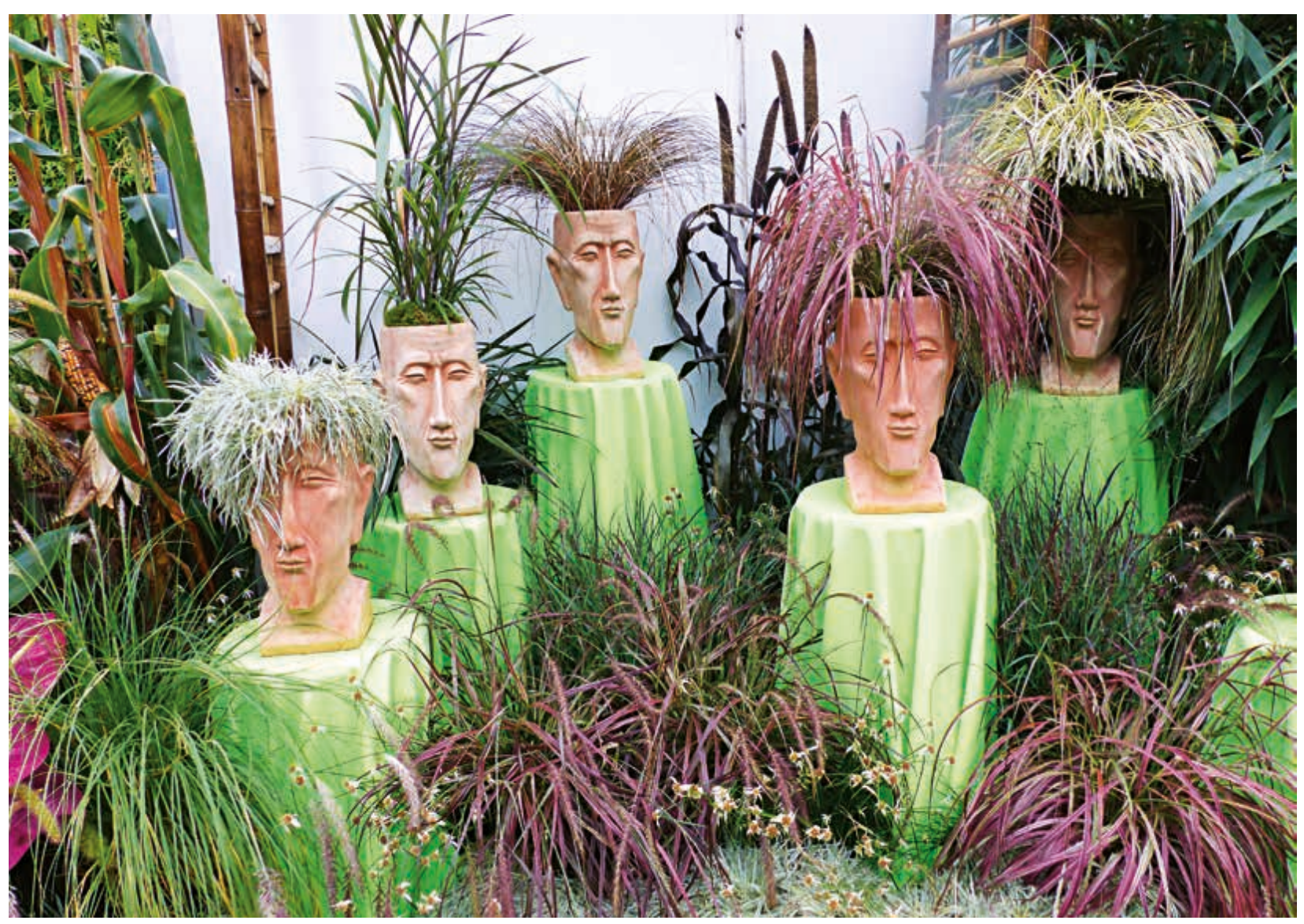

Abb. 12: Grasköpfe am Palmengarten-Stand während des Fürstlichen Gartenfestes auf Schloss Wolfsgarten. 


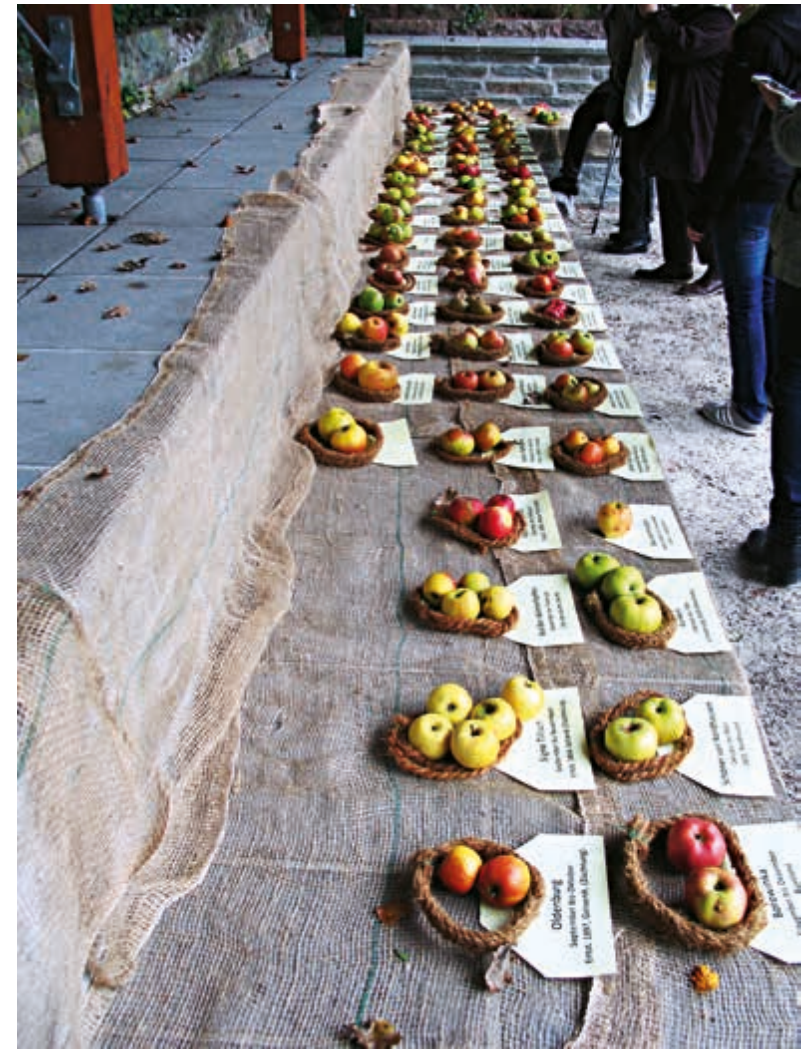

Abb. 13: Apfelschau im Oktober im Botanischen Garten.

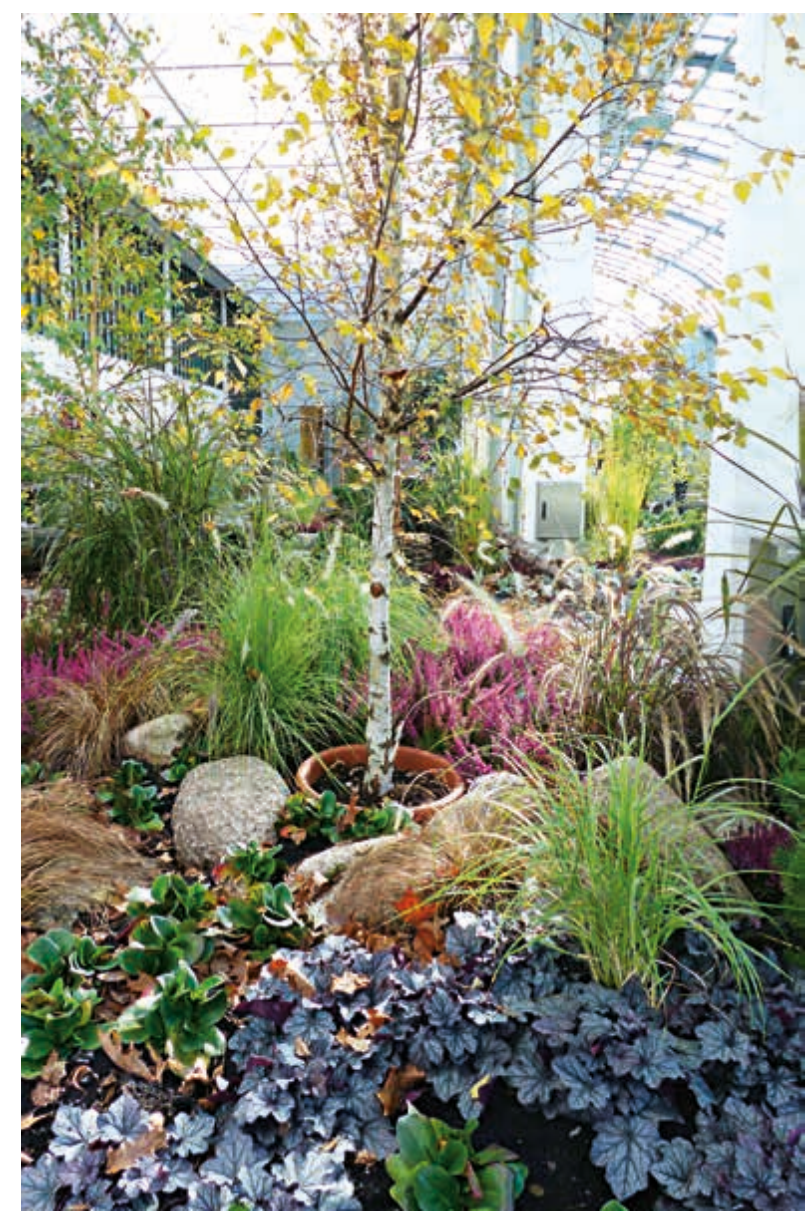

Abb. 14: Blumen des Herbstes in der Galerie West.
Kürbisse zu schnitzen. Zeitgleich mit dem Herbstfest präsentiert sich der Palmengarten jedes Jahr auf dem Fürstlichen Gartenfest auf Schloss Wolfsgarten in Langen. Für die Standgestaltung zum Thema "Gräser" gibt es als Pressepreis den silbernen Wolfskopf. Auch im Palmengarten gedeihen Gräser sehr gut. Noch bis zum Frost wirken die Pampasgräser mit ihren großen, weißen Blütenständen äußerst attraktiv.

Der „Tag der offenen Tür“ des Botanischen Gartens findet Anfang September zum 20. Mal statt. Ein breites Angebot an Pflanzen, Führungen und Aktionen, zusammen mit befreundeten jahrelangen Wegbegleitern wie z. B. dem NABU Frankfurt runden diesen wieder sehr gelungenen Tag ab. Viele Freundinnen und Freunde des Botanischen Gartens genießen auch das kulinarische Angebot, welches von aktiven Freundeskreis-Mitgliedern präsentiert wird. Bereits zum zweiten Mal lockt das Thema „Bienen in der Stadt" eine wiederum sehr große Zahl an Menschen in den Botanischen Garten - das „Frankfurter Bienenfestival“ ist auch in der Wiederholung ein großer Erfolg.

Von Oktober bis in den November hinein werden Pflanzenzeichnungen der Society of Botanical Arts ausgestellt. Das Motto „Den Pflanzen auf der Spur“ widmet sich den Pflanzenjägern, die weltweit unterwegs waren auf der Suche nach neuen exotischen Pflanzen. Zur Eröffnung kommt Floria Landgräfin von Hessen in den Palmengarten. So langsam müssen empfindliche Pflanzen wie das Mammutblatt mit entsprechendem Schutz winterfest gemacht werden. In der Galerie bringen die „Blumen des Herbstes“ noch einmal Farbe in die dunkle Jahreszeit. Chrysanthemen gibt es in dieser Saison weniger zu sehen, dafür zeigt sich eine in Anlehnung an die Natur gestaltete Heidelandschaft, in der auch herbstliches Falllaub nicht fehlt.

Auch die Abschlussveranstaltung des Jahres 2016 im Botanischen Garten, die den Reigen an Führungen, Aktionen und Sonderveranstaltungen beschließt, nämlich die Apfelschau, wird noch mal ein wunderbarer Termin. Eine unglaubliche Anzahl an besonderen und ausgefallenen Apfelsorten 


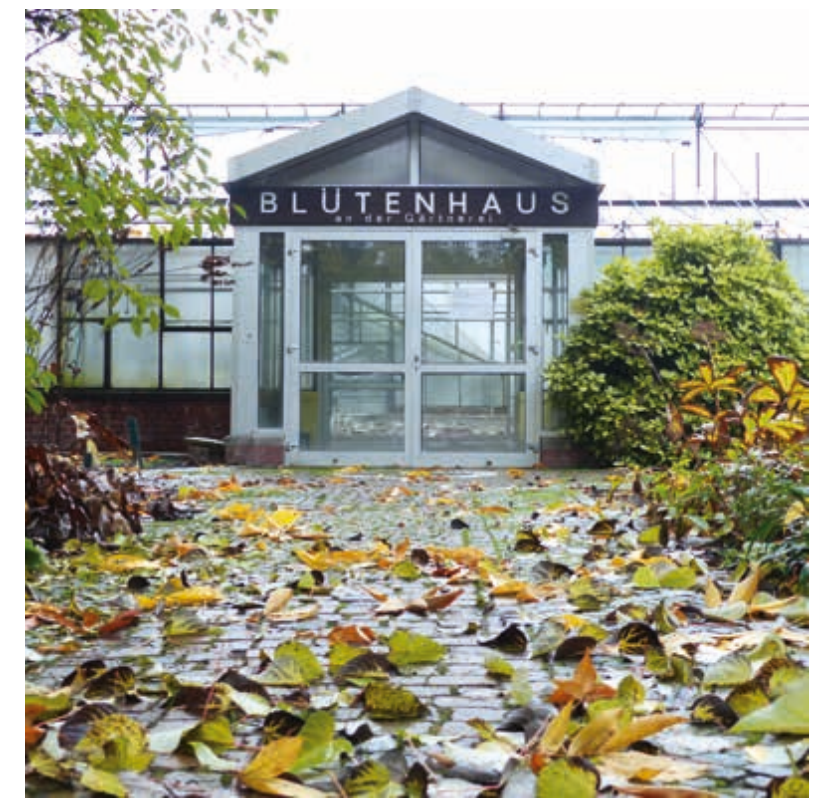

Abb. 15: Eine der letzten Aufnahmen des alten Blütenhauses vor dem Abriss zu Gunsten des neu zu bauenden Blütenund Schmetterlingshauses.

wird gezeigt. Auch die Verkostung und der Erwerb der Köstlichkeiten kommen zu ihrem Recht.

Am 1. November 2016 ist die Saison im Botanischen Garten wie gewohnt beendet und der Garten schließt seine Pforten. Die Gärtnerinnen und Gärtner sowie die Technische Leitung bereiten sich auf die bevor stehenden Winterarbeiten vor. Dazu gehören die Sanierung der Kieswege und des großen Sitzplatzes oberhalb des Alpinums, erste Baumaßnahmen im Rahmen unseres Projekts „Barrierefreiheit im Botanischen Garten“ sowie spezielle Baumarbeiten zum Erhalt unserer Gehölze. Viele Arbeiten warten auf Erledigung, damit das neue Gartenjahr 2017 beginnen kann.

Da die Vegetationsperiode länger geworden ist, sollte man mittlerweile eher von goldenem November statt von goldenem Oktober reden. Viele Bäume sind erst Anfang bis Mitte November richtig bunt gefärbt. Ein Hingucker ist die nordamerikanische Scharlach-Eiche mit ihren leuchtend rot-orangen Blättern am Oktogonbrunnen. Im Laufe der Zeit ist das Tropicarium in die Jahre gekommen und muss dringend für mehrere Mio. Euro saniert werden. Die Sanierung soll schrittweise in den nächsten drei Jahren geschehen. Sie ist nun vom Magistrat beschlossen und bewilligt.

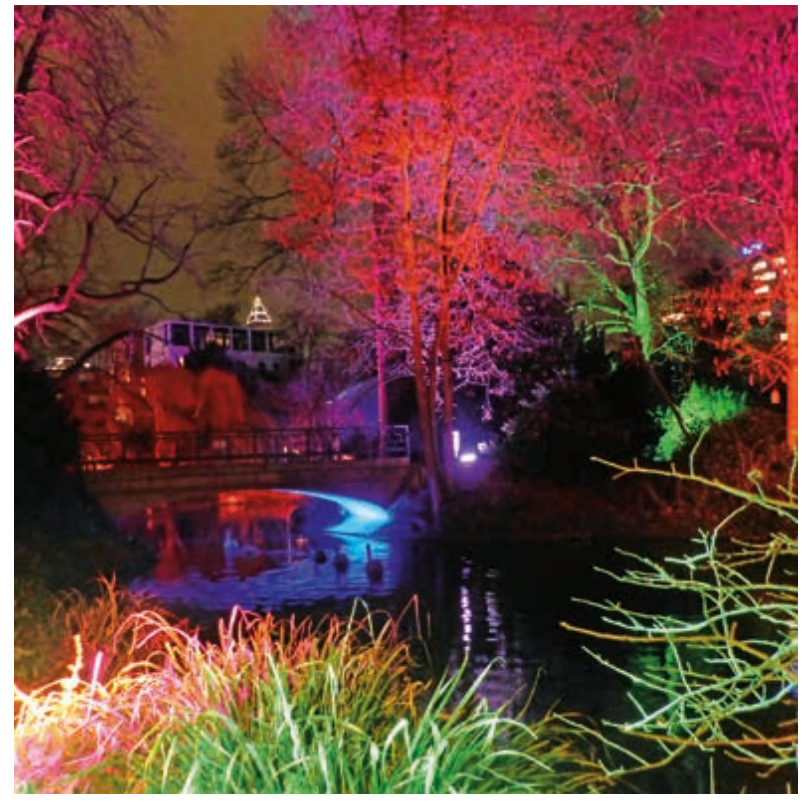

Abb. 16: Winterlichter am Kleinen Weiher.

Auch mit dem Bau des neuen Blüten- und Schmetterlingshauses soll es nun bald losgehen. Noch im November, pünktlich zum 1. Advent, wird die Weihnachtausstellung eröffnet. Ähnlich wie im Vorjahr bestehen Weihnachtsbaumschmuck und andere Dekorationen vor allem aus verarbeiteten echten Früchten, Zapfen und Zweigen.

Im Dezember werden nun schon zum vierten Mal die Winterlichter angeschaltet, die den Garten illuminieren. Eine neue LED-Technik lässt die Stämme der angestrahlten Bäume in intensiverem Licht erscheinen. Am ersten Tag der Winterlichter ist noch die alte Leas Eiche grün beleuchtet. Kurz danach wird der riesige Baum, der älter als der Palmengarten ist und aus der 1. Hälfte des 18. Jahrhunderts stammt, gefällt, weil er vom Pilz befallen, morsch und einsturzgefährdet ist. Die Gastronomie Goose verlässt kurzfristig die Villa Leonhardi. Für die Zeit der Winterlichter wird Ersatz gefunden, um den abendlichen Besuchern eine kleine Stärkung anzubieten.

Ein ereignisreiches Jahr ist vergangen, ein neues steht bevor, das u. a. durch viele Sanierungs-und Baumaßnahmen (Erneuerung der Wasser-Ringleitung, Renovierung des Tropicariums, Neubau des Blüten-und Schmetterlingshauses) geprägt sein wird. 